\title{
Application of single crystalline tungsten for fabrication of high resolution STM probes with controlled structure ${ }^{1}$
}

\author{
A. N. Chaika ${ }^{a}$, S. S. Nazin ${ }^{a}$, V. N. Semenov ${ }^{a}$, V. G. Glebovskiy ${ }^{a}$, S. I. Bozhko ${ }^{a, b}$, \\ O. Lübben ${ }^{\mathrm{b}}$, S. A. Krasnikov ${ }^{\mathrm{b}}$, K. Radican ${ }^{\mathrm{b}}$, I. V. Shvets ${ }^{\mathrm{b}}$ \\ ${ }^{a}$ Institute of Solid State Physics RAS, Chernogolovka, Moscow District, 142432, Russia \\ ${ }^{b}$ CRANN, School of Physics, Trinity College Dublin, Dublin 2, Ireland
}

\begin{abstract}
The possibility to fabricate scanning tunneling microscopy (STM) probes with controlled electronic structure using single crystalline tungsten tips is discussed. High resolution power of oriented single crystalline probes is demonstrated in atomic and subatomic resolution STM studies of silicon, gallium telluride and graphite surfaces. The possibility of controllable selection of the tungsten tip atom electron orbitals responsible for the surface imaging in STM experiments is demonstrated.
\end{abstract}

KEYWORDS: tungsten; scanning tunneling microscopy; electron orbital

\section{INTRODUCTION}

The invention of scanning tunneling microscopy (STM) allowed investigations of the local electronic structure of conducting surfaces with atomic resolution [1,2]. High spatial resolution of the method is based on exponential dependence of the tunneling current $I_{t}$ on the distance between the probe and the sample atoms (tunneling gap) leading to one order of magnitude decrease in the tunneling current with every $1 \AA$ increase in the gap spacing. Considering the role of the probe in experiments, this dependence means that more than $90 \%$ of the tunneling current can be collected by one atom at the apex. The precise knowledge of its electronic structure is crucial for qualitative interpretation of atomically resolved STM data and for further enhancement of the spatial resolution because the electronic structure of the apex atom can modify the electron tunneling conditions in different surface regions. In some cases, the apex orbital structure can also be visualized directly at small tunneling gaps [3-7]. The knowledge of the probe atomic and electronic structure is important for explanation of chemical and spin contrast in atomic scale STM images.

Application of oriented single crystalline tips with a priori known orientation of the apex axis appears to be one of the promising methods of fabrication of STM probes with predictable electronic structure. The main objective of this work is related to possibility to control the probe apex atomic and electronic structure and spatial resolution of STM using oriented single crystalline tips.

\section{MATERIALS AND METHODS}

In this work we used single crystalline tips with axes directed along the [001] crystallographic direction (W[001] probes) since tungsten atom at the apex of the ideally

1 This work was supported by the Russian Academy of Sciences, the Russian Foundation for Basic Research (projects 08-02-00612, 08-02-00006), grant of the President of the Russian Federation for support of leading scientific schools (NSh-4415.2010.2) and SFI E.T.S. Walton Programme. 
terminated tip should have four nearest neighbors in the second layer. This could provide higher stability of the probe at small distances between the tip and sample atoms. The main advantages of the $\mathrm{W}[001]$ probes in comparison with traditional polycrystalline probes (e.g., possibility to control the apex atomic structure and select the tungsten tip atom orbital collecting most of the tunneling current) are demonstrated in experiments on reconstructed $\mathrm{Si}(111)$ and $\operatorname{GaTe}\left(\begin{array}{lll}1 & 0 & -2\end{array}\right)$ surfaces and (0001) cleavage plane of highly oriented pyrolytic graphite (HOPG).

The experiments were carried out at room temperature in controllable ultra high vacuum (UHV) conditions using STM GPI-300. The base pressure in the STM chamber did not exceed $1 \times 10^{-8} \mathrm{~Pa}$. W[001] tips for the experiments were fabricated from oriented $0,5 \times 0,5 \times 10 \mathrm{~mm}$ single crystalline bars using electrochemical etching in $2 \mathrm{M} \mathrm{NaOH}$ solution. Scanning electron microscopy (SEM) images of two typical single crystalline $\mathrm{W}[001]$ probes after electrochemical etching are shown on Fig. 1, $a-c$ and $d-f$. The apex radius of curvature for both tips does not exceed 20-30 nm (fig. 1, c, f).

High resolution SEM images (fig. 1, $b, c, f$ ) reveal contaminations of the tip apexes that is not feasible for controllable high resolution experiments. For removal of the contaminants from the $\mathrm{W}[001]$ tips and preparation of clean apexes with minimal radius of curvature in the STM chamber, we used UHV tip preparation procedure consisting on electron beam flash heating at temperatures of $1000-1500^{\circ} \mathrm{C}$ and co-axial ion sputtering [9].

High resolution power of the W[001] tips after this UHV treatment was initially confirmed in experiments on the model $\mathrm{Si}(111) 7 \times 7$ reconstruction (fig. 2) and then in high resolution STM studies of the atomic structure of stepped $\mathrm{Si}(557) 5 \times 5$ surface and twocomponent GaTe( 10 -2) cleavage plane (fig. 3). The occupied electron state STM image of the $\operatorname{Si}(111) 7 \times 7$ surface shown in fig. 2, a (top panel) demonstrates features corresponding to the dangling bonds of the silicon atoms located in two topmost layers (surface layer adatoms and the second layer rest-atoms, fig. 2, a (bottom panel)). According to theoretical calculations [9], the simultaneous visualization of the electron states of the two topmost surface layer atoms can only be possible using sharp single atom terminated STM probe with minimal radius of curvature. High stability of the W[001] probes is proved by the minimal noise level on raw STM images of the $\mathrm{Si}(111)$ and GaTe(1 0 -2) surfaces shown in fig. 2 and 3.

\section{RESULTS AND DISCUSSION.}

STM images of the GaTe(1 0 -2 $)$ surface measured at different tunneling parameters (fig. 3) demonstrate selective imaging of the electron states corresponding to either tellurium (fig. 3, a) or gallium (fig. 3, b) atoms of the surface layer as well as simultaneous visualization of the electron states of both sublattices (fig. 3,c). The origin of the observed chemical selectivity is a subject of further investigations ${ }^{2}$, however, the high quality of the measured images demonstrates the excellent characteristics of the single crystal W[001] probes. Nevertheless, the main asset of the oriented single crystal tungsten probes is related to their predictable atomic and electronic structure.

A series of consecutive STM images of the $\mathrm{Si}(111) 7 \times 7$ surface shown in fig. $2, b$ (top panel) demonstrates this advantage of the crystallographically oriented tips. Several images from the sequence reveal uncontrollable changes of the W[001] tip state which were mainly observed at positive and small negative bias voltages that correspond to smaller distances between the tip and sample atoms in STM experiments. The switches of the tip

\footnotetext{
${ }^{2}$ Yashina L.V. Atomic geometry and electron structure of the $\operatorname{GaTe}\left(\begin{array}{lll}1 & 0 & -2\end{array}\right)$ surface / L.V. Yashina, R. Püttner, A.A. Volykhov [et al.] //. Phys. Rev. B. Submitted.
} 
state during scanning induce changes in the topography contrast and lateral shifts of the STM images by one lattice constant in the $\mathrm{W}\{011\}$ directions. Since the consecutive shifts are always oppositely directed and the surface atomic structure is not modified upon scanning, the observed effect can be explained by the apex atom jumps between two equivalent places on the apex of a non-ideal W[001] tip. From the knowledge of the tip orientation and the observed tip state switches, a realistic model of the probe atomic structure can be proposed (fig. 2, $c$ (bottom panel)). Note that even this simplified model can not be suggested for polycrystalline tungsten probe because the apex orientation after electrochemical etching and UHV tip treatment is unknown.

The knowledge of the $\mathrm{W}[001]$ probe electronic structure allowed us to realize controllable selection of the tip electron orbitals responsible for atomically resolved imaging in STM experiments on a graphite(0001) surface [10].

The regular two-fold and four-fold split subatomic features with shapes typical for $d_{x z}$ and $d_{x-y}^{2}{ }^{2}$ tungsten atomic orbitals (see fig. $4, b-g$ ), reproducibly observed in a number of experiments, prove principal possibility of the preferable electron tunneling through particular orbitals of the tungsten tip atom. For the W[001] probe-graphite(0001) surface system (fig. $4, a$ ) this was achieved by a precise choice of the bias voltage and tip-sample distance. The observed features are explained by decisive contribution of $d_{3 z}{ }^{2}{ }^{2}$ (fig. $4, b$ and $e$ ), $d_{x z, y z}$ (fig. 4, $c$ and $f$ ) and $d_{x-y}^{2}$ (fig. 4, $d$ and $g$ ) electron states of the W[001] tip. To realize the tunneling conditions shown in fig. $5, b$, the bias voltage in the experiments was adjusted in accordance with the partial density of electron states (PDOS) of the W[001] tip atom $n_{t}$ calculated in frames of the tight binding model (TB, see the bottom panel of fig. 5, $a)$. The density of electron states near the Fermi level in fig. 5, $b$ both for the tip $\left(n_{t}(E)\right)$ and sample $\left(n_{s}(E)\right)$ atoms are drawn in accordance with the results of the TB calculations. To reach maximum contribution of the $d_{3 z}{ }^{2}{ }_{-r}^{2}$ tip states, STM images were measured at small positive bias voltage between the sample and the tip (the voltage was applied to the sample while the tip was grounded). In this case, the highest spatial resolution could be achieved in STM experiments that was confirmed by the smallest diameters of the measured atomic features (fig. $4, b)$. At small negative bias voltages of $-(30-150) \mathrm{mV}$ and very small tipsample distances, the tunneling conditions corresponding to the surface imaging by the $d_{x}^{2}$. $y^{2}$ electron states of the tip could be achieved. This is confirmed by the regular four-fold splitting of all atomic features in the STM images (fig. 4, $d$ ). As illustrates the magnified view of the features in fig. $4, g$, the shape of the experimentally resolved subatomic features qualitatively agrees with spatial distribution of the $5 d_{x-y}^{2}{ }^{2}$ electron states in the tungsten atom. At intermediate tip-sample distances and small negative bias voltages, the two-fold split subatomic features with the shapes and dimensions typical for the $5 d_{x z}$ tungsten atomic orbital could be resolved. Three possibilities of the W[001] tip atomic orbital selection at different tip-surface separations are schematically shown in fig. 4, $a$.

The models in fig. 4, $a$ and $5, b$ do not include the interaction between the tip and sample atoms which is essential at small tunneling gaps. As demonstrate the density functional theory (DFT) calculations of the PDOS associated with different electron orbitals of the tungsten apex atom (fig. 5, a), a decrease in the tip-sample distance leads to gradual depopulation of the atomic orbitals further extended along the tip axis $\left(d_{3 z-r}{ }^{2}\right.$ and then $d_{x z, y z}$ ) because of their overlapping with carbon electron orbitals of the graphite surface atoms. The depopulation of the $d_{3 z}{ }^{2}{ }^{2}$ orbital with decrease of the gap spacing from 4 to $2-2,5 \AA$ (fig. $5, a$ ) stipulates an increase of the relative contribution to the tunneling current of the $d_{x z, y z}$ and $d_{x-y}^{2}{ }^{2}$ tip orbitals. As a result, STM images measured at small tipsample distances can reveal asymmetric features due to decisive contribution of the tungsten tip atom $d$-electron states with non-zero orbital momentum projection on the $z$ axis $\left(d_{x z, y z}, d_{x y}\right.$ and $\left.d_{x-y}^{2}\right)$. The tip-sample distance dependence of the graphite surface STM images measured at fixed bias voltage of $-35 \mathrm{mV}$ is shown in fig. $6, a$. The observed change of the symmetry of the subatomic features with decreasing gap spacing (increasing 
tunneling current) agrees with theoretical spatial distribution of $d$-orbitals with different orbital momentum projections on the z-axis. Figures $6, b$ and $6, c$ show that the shapes of the experimentally measured subatomic features are in good agreement with theoretical electron density distribution maps of the $\mathrm{W}[001]$ tip atom interacting with the graphite surface calculated using DFT method (fig. $6, b$ ). In this case different electron $d$-orbitals of the tungsten tip atom are probed by further extended $s$ - and $p$-orbitals of the graphite surface carbon atoms at different tip-sample distances. However, the situation can be reversed for another tip-sample system. In particular, different spatial distribution of the $\mathrm{Ga} 4 p$ and Te5p-orbitals and distance dependent contribution of $s$ - and $p$-orbitals of gallium and tellurium atoms can be responsible for the observed chemical selectivity in STM images of the GaTe( $\left.\begin{array}{lll}1 & 0 & -2\end{array}\right)$ cleavage plane measured at the same bias voltage (fig. 3).

\section{CONCLUSIONS}

We have demonstrated principal possibilities to control electron orbital structure of STM probes using oriented single crystalline tips and selectively probe different orbitals of the surface atoms at precise choice of the tip, bias voltage and tunneling gap between interacting tip and surface atoms. The results of the work are very important for further enhancement of the spatial resolution of STM, better understanding of the STM image formation for multi-component systems and controllable chemical analysis of the surfaces at the atomic scale.

\section{REFERENCES}

1. Binnig G., Rohrer H. // Scanning tunnelling microscopy. Helv. Phys. Acta, 1982, vol. 55, p. $726-735$.

2. Binnig G., Rohrer H., Gerber Ch., Weibel E. // Surface studies by scanning tunneling microscopy. Phys. Rev. Lett., 1982, vol. 49, p. 57-61.

3. Herz M., Giessibl F.J., Mannhart, J. // Probing the shape of atoms in real space. Phys. Rev. B, 2003, vol. 68, p. 045301(1-7).

4. Zotti L.A., Hofer W.A., Giessibl F.J. // Electron scattering in scanning probe microscopy experiments. Chem. Phys. Lett., 2006, vol. 420, p. 177-182.

5. Chaika A.N., Semenov V.N., Nazin S.S., et. al. // Atomic row doubling in the STM images of $\mathrm{Cu}(014)-\mathrm{O}$ obtained with $\mathrm{MnNi}$ tips. Phys. Rev. Lett., 2007, vol. 98, p. 206101(1-4).

6. Murphy S., Radican K., Shvets I.V., et. al. // Asymmetry effects in atomically resolved STM images of $\mathrm{Cu}(014)-\mathrm{O}$ and $\mathrm{W}(100)-\mathrm{O}$ surfaces measured with MnNi tips. Phys. Rev. B, 2007, vol. 76, p. 245423(1-16).

7. Chaika A.N., Myagkov A.N. // Imaging atomic orbitals in STM experiments on a Si(111)-(7×7) surface. Chem. Phys. Lett., 2008, vol. 453, p. 217-221.

8. Chaika A.N., Semenov V.N., Glebovskiy V.G., Bozhko S.I. // Scanning tunneling microscopy with single crystalline W[001] tips: high resolution studies of $\operatorname{Si}(557) 5 \times 5$ surface. Appl. Phys. Lett., 2009, vol. 95, p. 173107(1-3).

9. Wang Y.L., Gao H.-J., Guo H.M., et. al. // Tip size effect on the appearance of a STM image for complex surfaces: Theory versus experiment for Si(111)-(7×7). Phys. Rev. B, 2004, vol. 70, p. 073312(1-4).

10. Chaika A.N., Nazin S.S., Semenov V.N., et. al. // Selecting the tip electron orbital for scanning tunneling microscopy imaging with sub-Ångström lateral resolution. EPL, 2010, vol. 92, p. 46003(1-6). 

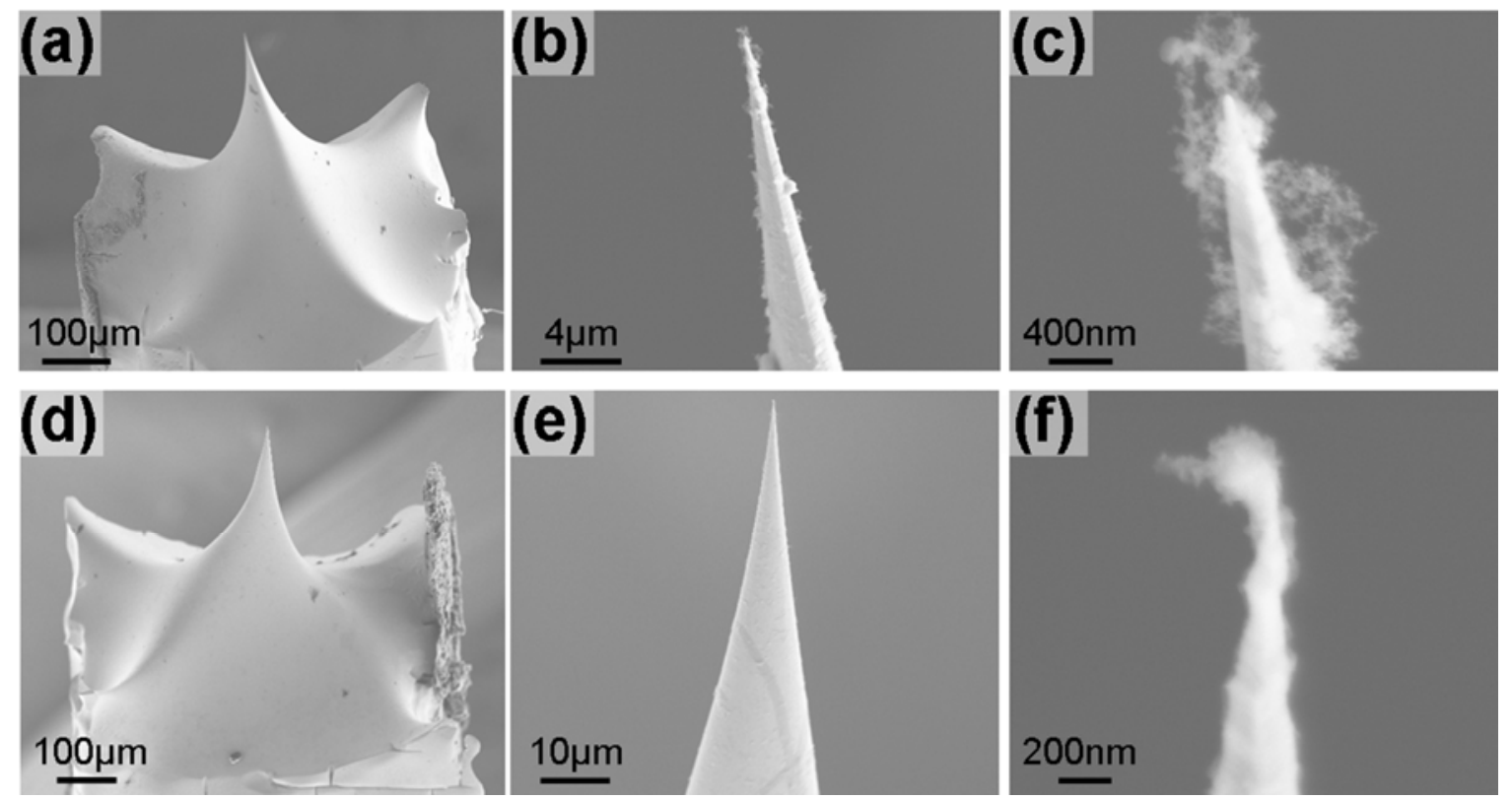

Fig. 1. SEM images of two single crystalline W[001] tips ( $a-c$ and $d-f$, respectively) after electrochemical etching.
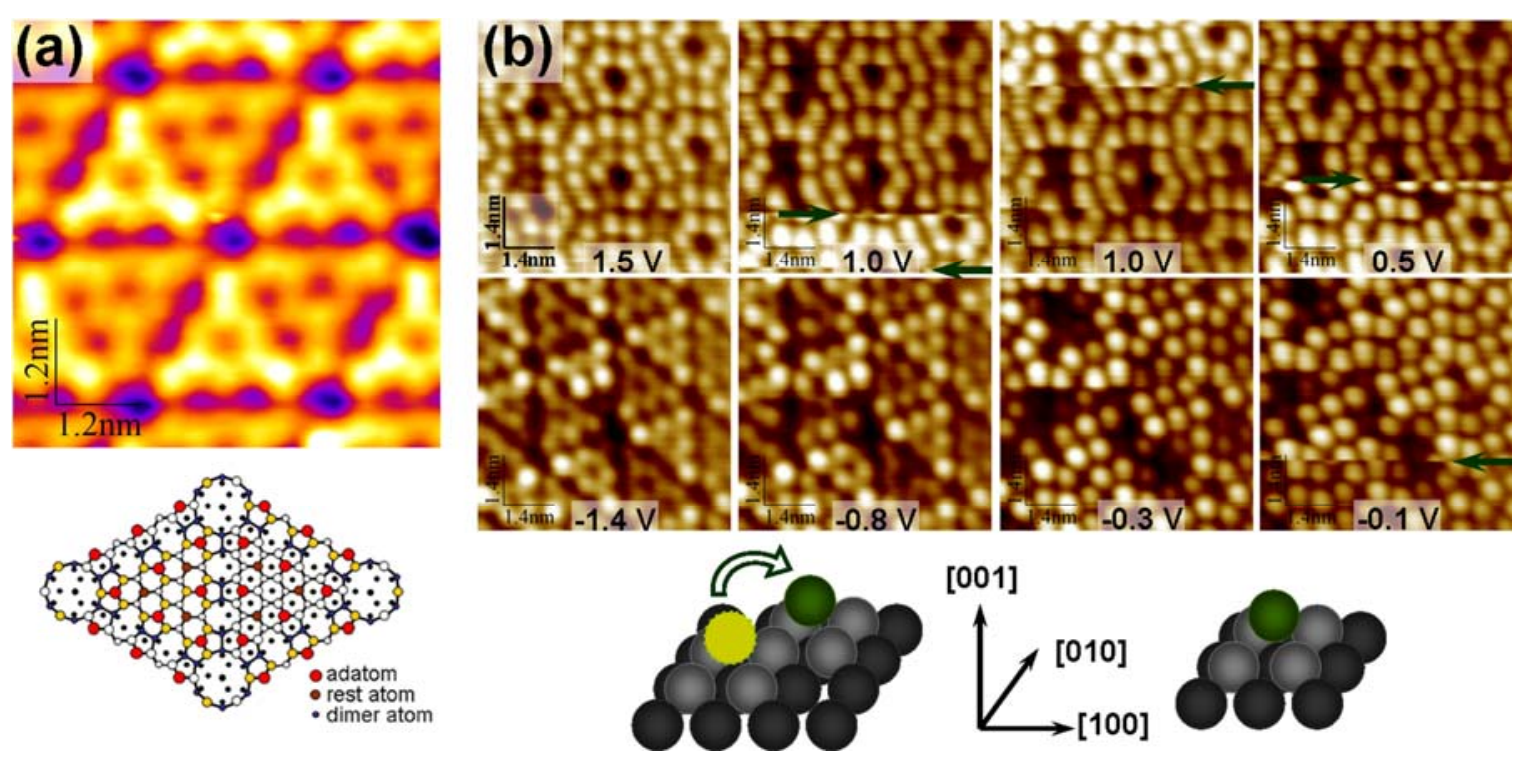

Fig. 2. (a) A schematic model of the unit cell (bottom panel) and occupied state STM image of the $\operatorname{Si}(111) 7 \times 7$ surface measured with a $\mathrm{W}[001]$ probe at $U=-1.4 \mathrm{~V}$ and $I_{t}=50$ pA (top panel). (b) STM images of the $\operatorname{Si}(111) 7 \times 7$ surface taken at tunneling current $I_{t}=$ $40 \mathrm{pA}$ and bias voltages of 1,$5 ; 1,0 ; 0,5 ;-1,4 ;-0,8 ;-0,3 ;-0,1 \mathrm{~V}$ (top panel, horizontal arrows indicate the changes of the tip state inducing shifts of the images in $<011>$ directions) and a model of the atom jumps (bottom panel) between two equivalent positions at the apex of non-ideal $\mathrm{W}[001]$ probe [8] inducing the observed image shifts. 

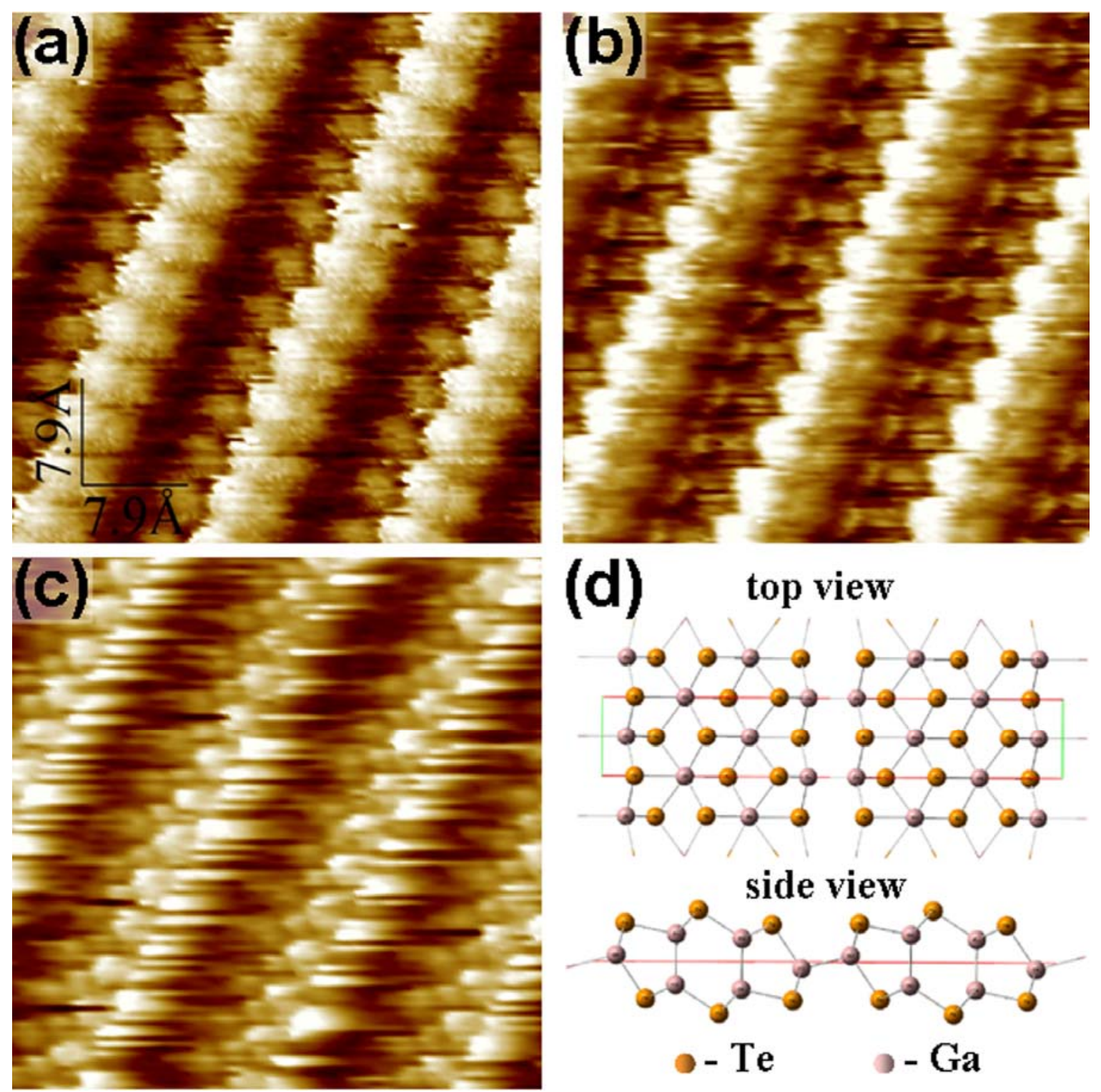

\section{top view}
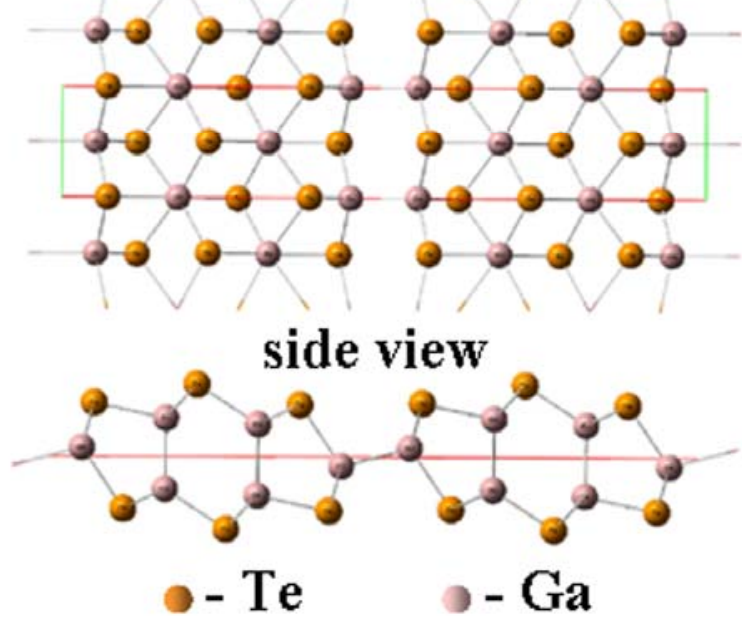

Fig. 3. $4 \times 4 \mathrm{~nm}$ STM images $(a-c)$ of the $\operatorname{GaTe}\left(\begin{array}{lll}1 & 0 & -2\end{array}\right)$ cleavage plane measured using $\mathrm{W}[001]$ tip (tunneling parameters: (a) $-U=-1,0 \mathrm{~V}, I_{t}=120 \mathrm{pA}$; (b) $-U=-1,0 \mathrm{~V}, I_{t}=50$ $\left.\mathrm{pA} ;(c)-U=-0,9 \mathrm{~V}, I_{t}=50 \mathrm{pA}\right)$ and schematic model of the GaTe(1 $\left.0-2\right)$ surface $((d)-$ top and side views). 

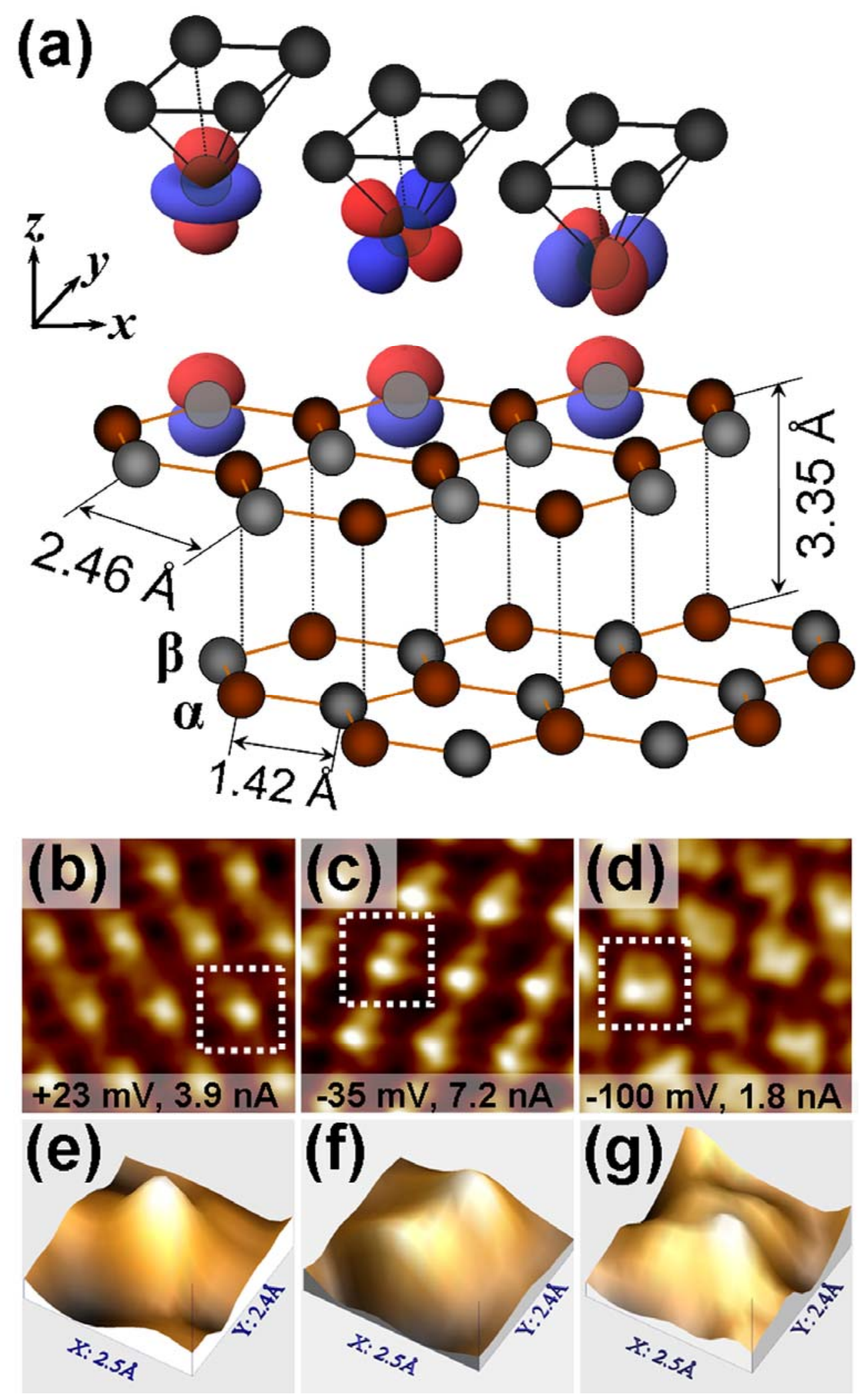

Fig. 4. (a) Schematic model of the W[001] probe - graphite surface system. (b-d) STM images of the graphite surface measured with the $\mathrm{W}$ [001] probe (tunneling parameters: $(b)$ $-U=-23 \mathrm{mV}, I_{t}=3,9 \mathrm{nA}$; (c) $-U=-35 \mathrm{mV}, I_{t}=7,2 \mathrm{nA}$; (d) $-U=-0,1 \mathrm{~V}, I_{t}=1,8 \mathrm{nA}$; $(e-$ g) $3 \mathrm{D}$ images of individual atomic features were taken from the $2 \times 2 \AA$ regions indicated in panels $(b-d)$. 

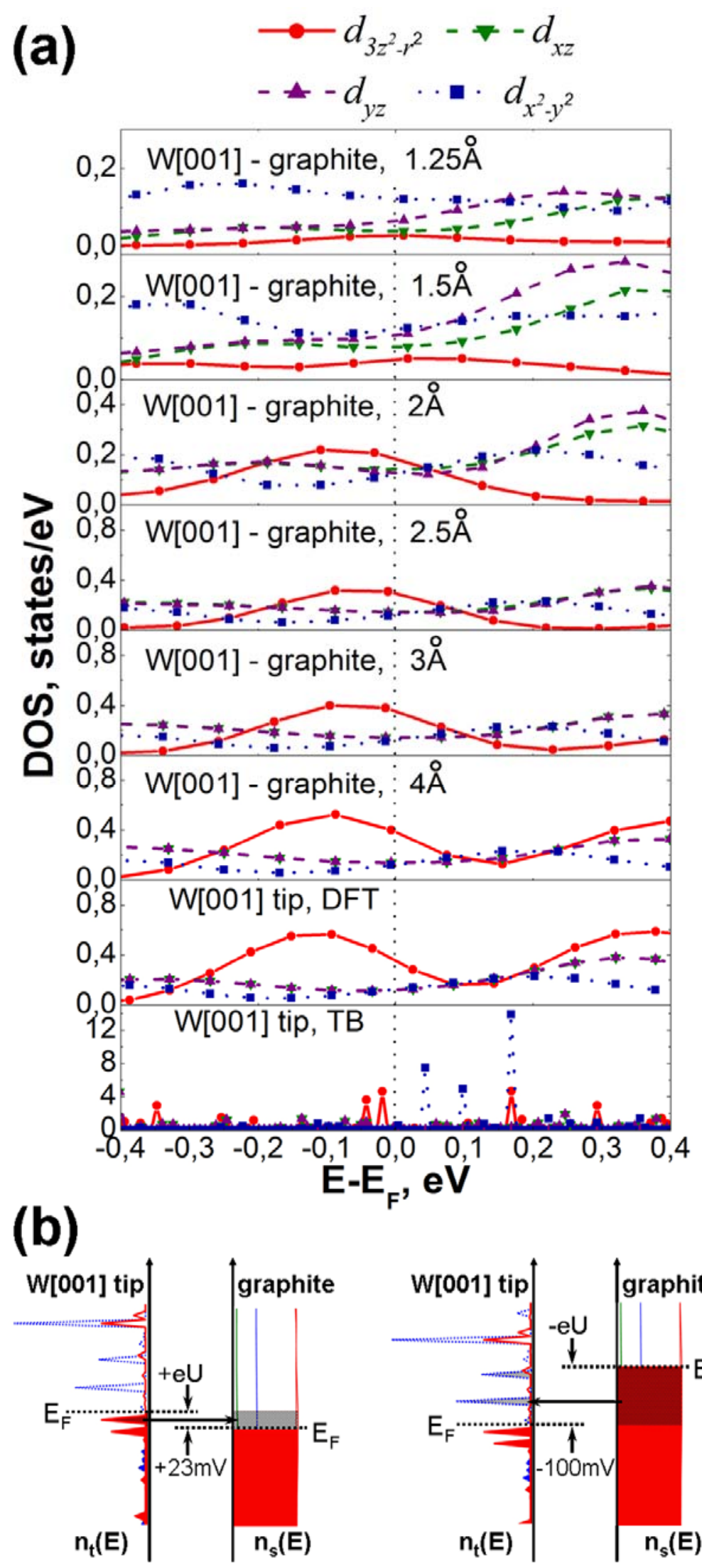

Fig. 5. Partial density of electron $d$-states associated with the apex atom of the W[001] tip calculated in frame of the density functional theory (DFT) and TB method (a) at different distances between interacting W[001] tip and graphite(0001) surface atoms (the tunneling gaps in Ångströms are indicated on the corresponding panels). Schematic model of the tunneling process in the occupied (right) and empty (left) surface state regimes (b). 

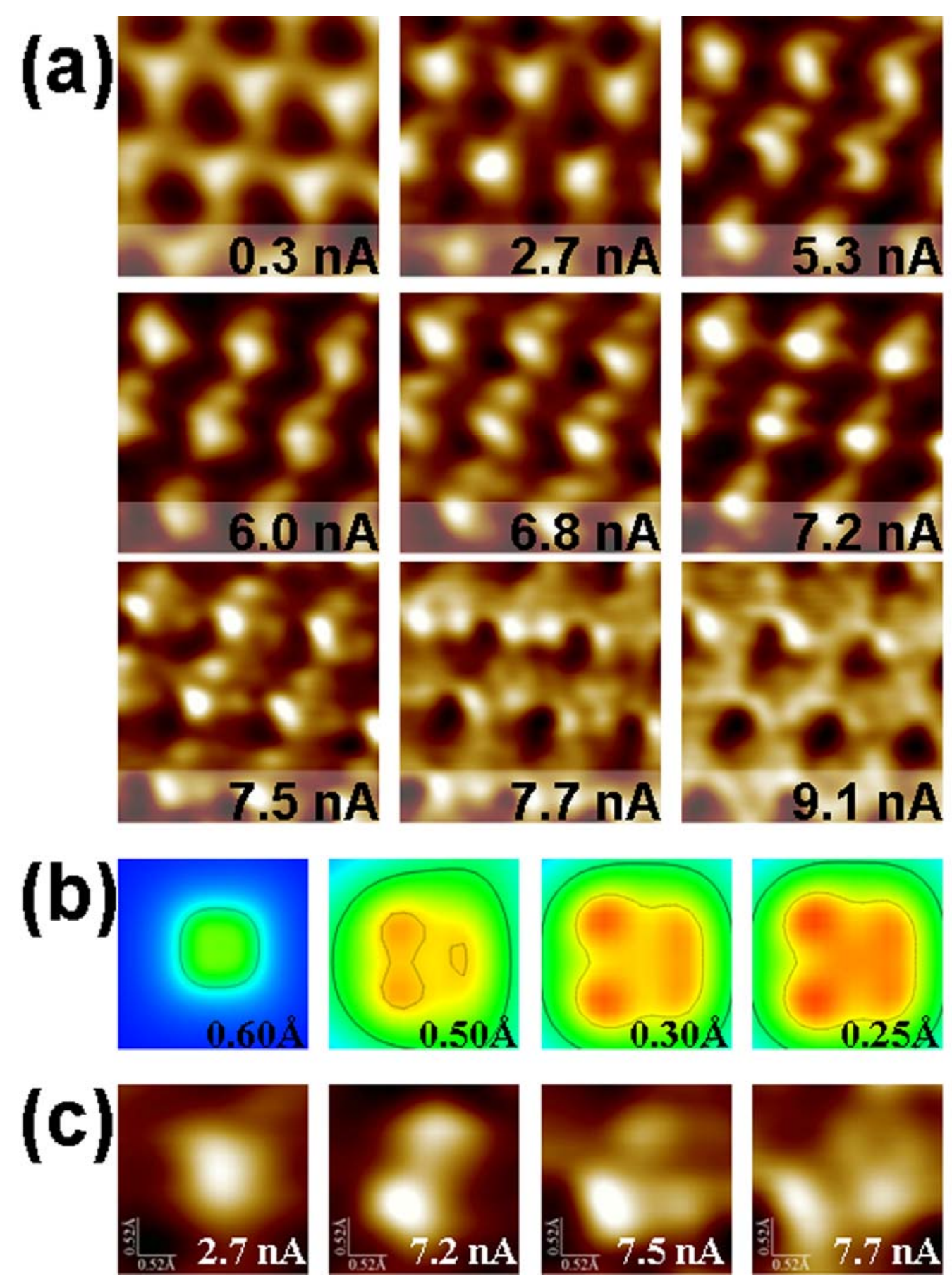

Fig. 6. (a) $7 \times 7 \AA \mathrm{STM}$ images of the graphite(0001) surface as a function of tip-sample distance (the change of the distance was achieved by varying the tunneling current from 0,3 to $9,1 \mathrm{nA}$ at unchanged $\mathrm{W}[001]$ tip state and fixed bias voltage of $-35 \mathrm{mV}$ ). (b) calculated electron density distribution maps shown as slices by (001) planes located on different distances from the tungsten atom at the apex of the W[001] tip interacting with the carbon atom of the graphite surface (the distances from the tungsten atom core to the (001) plane are shown on each particular frame; the core-core distance between interacting tip and sample atoms is $1,75 \AA$ ). (c) Magnified views of the $2,1 \times 2,1 \AA$ regions of the experimental STM images in panel $a$. 